\title{
Bericht über die kroatisch-österreichische Sommerschule Verflechtungen und Interferenzen im zentraleuropäischen Raum in Labin
} Václava Beyerová

Zwischen dem 20. und 24. September 2017 fand in der kroatischen Stadt Labin die kroatisch-österreichische Sommerschule mit dem Thema Verflechtungen und Interferenzen im zentraleuropäischen Raum statt. Diese Sommerschule entstand dank der Organisation der Philosophischen Fakultät der Universität Zagreb und der Universität Wien unter Mitarbeit vom österreichischen Kulturforum Zagreb und den Kulturinstitutionen in Labin. Sie setzte sich als Ziel, Geschichte und Gegenwart des zentraleuropäischen Raumes unter kulturwissenschaftlichen Perspektiven zu erforschen. Zu den besprochenen Themen, die das Konzept des Programms bildeten, gehörten: Zentraleuropa als pluri-kultureller Raum von Ähnlichkeiten und Differenzen, die Narrativität von Identitäten, das Verhältnis von Zentrum und Peripherie, Postimperialismus und Postkolonialismus und die Wechselwirkungen zwischen unterschiedlichen Literaturen.

Am ersten Tag nach der offiziellen Eröffnung der Sommerschule durch die Organisatoren Wolfgang Müller-Funk, Marijan Bobinac und den Bürgermeister der Stadt Labin Zoran Rajković begann das Programm, das als viertägiger intensiver Workshop konstruiert wurde. Es bestand aus täglichen Einführungsvorträgen von Zagreber und Wiener Wissenschaftlern, nach denen die beteiligten Studierenden mit den daran anknüpfenden literarischen Texten, Essays und theoretischen Aufsätzen in Gruppen arbeiteten. Das akademische Team, das diese Veranstaltung betreute setzte sich aus Dozentinnen und Dozenten aus Wien (Wolfgang Müller-Funk, Andrea Seidler, Clemens Ruthner, Anna Babka, Johanna Chovanec) und Zagreb (Marijan Bobinac, Svjetlan Lacko Vidulić, Jelena Spreicer), die zwar seit mehreren Jahren mitarbeiten, aber diesmal auch im Rahmen des Forschungsprojektes „Postimperiale Narrative in den zentraleuropäischen Literaturen der Moderne“, zusammen.

Wolfgang Müller-Funk und Clemens Ruthner beleuchteten im Rahmen des ersten theoretischen Beitrags die Frage der Liminalität und Imagines, wobei erklärt wurde, was (sichtbare und unsichtbare) Grenzen sind und was sie konstituieren. Im Zusammenhang mit der Imagologie richteten sie das Hauptaugenmerk auf die Erklärung der Form von 
Bildern des Eigenen und Fremden in Bezug auf die Liminalität, die einen gewissen Übergang bezeichnet. Weiter Vorträge waren z. B. von P. Žagar-Šoštarić $O$. Gross und Nordadria, H.-P. Kucher: „Triste Trieste allegra.../ tristes vergnügtes Triest... “ Zwischen „scontrosa grazia/ sprödem Charme“ und „scritture parallele“ - Blicke auf und in die Literatur aus Triest im 20. Jahrhundert oder von J. Chovanec und von W. Müller Funk: Der Osmanische und Habsburgische Mythos. Zu den Texten, die im Anschluss an die Vorträge behandelt wurden, gehörten zum Beispiel Werke von Peter Altenberg, Hermann Bahr, Miroslav Krleža, Robert Musil, Heimito von Doderer, Peter Handke und Joseph Roth.

Da der Untertitel dieser Sommerschule Wissenschaft, Literatur, Kunst, Film $\mathcal{E}^{2}$ Musik ist, wurden neben den Vorträgen und dem Close Reading, die vormittags auf dem Programm standen, die Nachmittage auch dem künstlerischen Team gewidmet. Gleich am ersten Tag der Sommerschule fand die Vernissage der modernen österreichischen Künstlerin Barbara Höller statt, die von Sabine Müller-Funk kuratiert wurde. In den nächsten Tagen gab es auch eine Filmnacht mit dem Film Untitled von Michael Glawogger, ein Konzert und eine Lesung des österreichischen Schriftstellers Norbert Gstrein (Das Handwerk des Tötens, 2003; Die Winter im Süden, 2008).

Die Teilnehmer der Brünner Germanistik, die auch die Gelegenheit hatten, an dieser Sommerschule teilzunehmen, konnten dadurch neue Erkenntnisse bezüglich der besprochenen Themen gewinnen. Die folgenden Diskussionen mit den Wissenschaftlern halfen nicht nur beim Begreifen all der Themen, sondern auch bei ihrer zukünftigen wissenschaftlichen Arbeit.

Schließlich sollte noch betont werden, dass die präzisen Vorbereitungen des Organisationsteams die Erwartungen aller Teilnehmer übertrafen und dass sich diese nicht nur auf die hoffentlich weiteren Jahrgänge dieser Sommerschule, sondern auch auf mögliche weitere Kooperationen, bereits freuen.

Václava Beyerová / 450237@mail.muni.cz

Masarykova univerzita, Filozofická fakulta, Ústav germanistiky, nordistiky a nederlandistiky Arne Nováka 1, 60200 Brno, CZ 